\title{
Field-based development of an information support robot for persons with dementia
}

\author{
Takenobu Inoue $^{\mathrm{a}, *}$, Misato Nihei ${ }^{\mathrm{b}}$, Takuya Narita ${ }^{\mathrm{b}}$, Minoru Onoda $^{\mathrm{b}}$, Rina Ishiwata ${ }^{\mathrm{a}}$, Ikuko Mamiya ${ }^{\mathrm{a}}$, \\ Motoki Shino $^{\mathrm{b}}$, Hiroaki Kojima ${ }^{\mathrm{c}}$, Shinichi Ohnaka ${ }^{\mathrm{d}}$, Yoshihiro Fujita ${ }^{\mathrm{d}}$ and Minoru Kamata ${ }^{\mathrm{b}}$ \\ ${ }^{a}$ Research Institute, The National Rehabilitation Center for Persons with Disabilities, Tokorozawa, Japan \\ ${ }^{\mathrm{b}}$ Graduate School of Engineering, The University of Tokyo, Tokyo, Japan \\ ${ }^{\mathrm{c}}$ National Institute of Advanced Industrial Science and Technology, Tsukuba, Japan \\ ${ }^{\mathrm{d}}$ NEC Corporation, Tokyo, Japan
}

\begin{abstract}
Background: The use of robots for providing support to persons with dementia is very promising. However, it is difficult to develop a truly useful robot system because of the difficulty of clarifying their needs and opinions.

Objective: Because a field-based method is effective for the development of these kinds of systems, in this study, we developed a prototype of an information support robot for persons with dementia, using field-based methodology.

Methods: A communication robot system produced by NEC Corporation, called "PaPeRo", was chosen as the platform for this system. To achieve the goal of keeping persons with dementia informed of their daily schedule and prompting them to take desired actions, interactive verbal communication algorithms were programmed into the robot system.

Results: The results of experiments with five dementia sufferers showed that this system produced an information acquisition rate of over $90 \%$. In addition, a life support demonstration showed the possibility of prompting users to perform actions.

Conclusions: These results suggest the possibility of using this robot to support independent living by persons with dementia, and also the effectiveness of the field-based method.
\end{abstract}

Keywords: Assistive technology, field-based innovation, development methodology, communication robot, verbal communication, assistive robots

\section{Introduction}

The number of persons with dementia is increasing worldwide, and it has been estimated that the figure will reach 65.7 million in 2030 and 115.4 million in 2050 [1]. In particular, estimates have shown that the number in Japan will reach 3.2 million by 2025 [2]. More specifically, the number of persons with mild dementia is predicted to rise markedly as a result of medical progress that can slow the course of the disease, and advances in diagnostic technologies that will facilitate its early detection. Based on these perspectives, there are greater expectations from assistive technologies to

* Corresponding author: Takenobu Inoue, Director, Department of Assistive Technology, Research Inst., NRCD, Namiki 4-1, Tokorozawa, Saitama 359-8555, Japan. E-mail: inoue-takenobu@rehab.go.jp. support persons with mild dementia, owing to the insufficient number of caregivers. For this reason, it is also important for these persons to be able to maintain their autonomy and independent living. Assistive technologies have a high potential to achieve these goals.

For some time now, advances have been made in robotic technologies, to the point that robots have a high potential for assisting the elderly [3]. There are some difficulties in developing a truly useful system for dementia sufferers, however, because most of them find it hard to express their thoughts, needs, and preferences. For this reason, subjective evaluation is not particularly effective in matching needs and technologies. Traditionally, robotic technology has progressed based on technology-oriented development. This has led many people in the field of assistive technology to claim that there are many things that a robot can do, 
but nothing of practical value for this application has been developed yet. Consequently, matching the needs of persons with dementia and robotic technology is a demanding challenge.

Several projects have been undertaken to develop different types of assistance for persons with dementia that employ information technologies [4-11]. One concept that has emerged in these projects is the importance of a good relationship between the development side and the user side. In this regard, the COGKNOW project (2006-2009) in the EU 6th framework program was quite unique, because it was run according to a comprehensive iterative project implementation plan to develop an information technology system for persons with mild dementia [12]. Their technical development process was split into three phases: mock-up development, prototype development, and stable prototype development. Field tests were conducted following each phase of development. Meanwhile, Inoue et al. proposed a field-based method for the development of assistive technologies for persons with dementia, and suggested its effectiveness in the development of a daily-plan indicator they could use [13]. This method is based on a concept similar to that of the COGKNOW project, but it targets an earlier stage of development. In this methodology, the importance of developing assistive technologies in the area of daily life for persons with dementia in the first stage was emphasized. Researchers went on the field, ascertained people's needs, and conducted an evaluation loop with a mock-up and prototype. This study showed that the field-based method was useful for determining the specifications of the daily-plan indicator being developed.

The purpose of the present study was to confirm the field-based method through the development of an information support robot for persons with dementia. In this study, the prototype of such a robot was developed based on the field-based method. A communication robot called "PaPeRo", which is manufactured by NEC Corporation, was adopted as the platform of this system, and information support algorithms for persons with dementia were programmed into the robot. This particular technology was chosen so as to provide confirmation because this robot has many functions, and matching these functions with the needs of persons with dementia is more important than the use of other technologies. This first prototype was installed in the home of a person with dementia who was willing to cooperate with our project. The information support algorithms were then modified according to the results of the evaluation. Next, the efficacy of a second prototype was evaluated based on evaluation experiments conducted with five persons with dementia in their own homes. Finally, the efficacy of the field-based method was discussed in terms of the process of the development of this robot system and its effectiveness.

\section{System concept of information support robot}

\subsection{Problems posed by information disabilities of dementia}

Problems related to memory and attention span that are common to those with dementia create many problems in their daily life. Since days, dates, time, and daily schedules are important information, a lack of awareness of this information causes many difficulties in their daily activities. For some persons with dementia, calendars, clocks, memo pads, and various other tools can be useful. Those with more severe dementia, however, pay no attention to these tools, and thus do not access the information they need. For example, if one cannot remember the time one goes to the daytime nursing home, he/she will not make the necessary preparations before leaving home. As a result, one may not be properly dressed, or may experience incontinence while on the courtesy bus to the nursing home because he/she did not go to the bathroom. This kind of information support is thus important and necessary in assisting persons with dementia.

\subsection{The communication robot "PaPeRo"}

Figure 1 shows the communication robot called " $\mathrm{Pa}$ PeRo" (Partner Personal Robot) [14]. The robot is equipped with speech recognition, speech synthesis, facial image recognition, autonomous mobility, head motion, light indication functions, and tactile sensors. Table 1 presents the specifications of the robot, which is currently used mainly as a research platform and for entertainment.

\subsection{Concept of information support robot system}

Figure 2 shows the concept of the proposed information support robot, whose key functions are as follows:

- To provide required information to users

- To prompt actions/activities in users

- To attract the attention of users

- To communicate with users through interactive conversation

We adopted "PaPeRo" as the platform for this system because its functions offer an advantage in combining these functions. 
Table 1

Specifications of the communication robot "PaPeRo"

\begin{tabular}{|c|c|}
\hline Dimensions & $\begin{array}{l}\text { Height: } 385 \mathrm{~mm} \\
\text { Width: } 282 \mathrm{~mm} \\
\text { Depth: } 251 \mathrm{~mm}\end{array}$ \\
\hline Weight & Approximately $6.5 \mathrm{~kg}$ \\
\hline Wheels & $\begin{array}{l}\text { It has a total of three wheels: two in front and one in back. The two front wheels are driven by two motors. The } \\
\text { maximum speed of movement is } 20 \mathrm{~cm} / \mathrm{s} \text {. }\end{array}$ \\
\hline Head & Moves up and down, and turns left and right with two motors. \\
\hline CCD camera & $\begin{array}{l}\text { Using CCD camera embedded in both of its eyes, it walks around objects, measures distances and approaches people, } \\
\text { follows people, remembers people, and differentiates among them. }\end{array}$ \\
\hline $\begin{array}{l}\text { Sound direction de- } \\
\text { tection microphone }\end{array}$ & $\begin{array}{l}\text { There are three microphones: one on the front, and one each on the left and right. PaPeRo estimates the direction of } \\
\text { sounds by means of the difference in time for sound to arrive at the respective microphones, and it looks back in the } \\
\text { direction of the sound. }\end{array}$ \\
\hline $\begin{array}{l}\text { Speech recognition } \\
\text { microphones }\end{array}$ & $\begin{array}{l}\text { One directional microphone is installed in the head. PaPeRo speaks while facing a person by means of image } \\
\text { recognition, and thus, the microphone points toward the person as well. There is another microphone for noise } \\
\text { cancellation, which enables PaPeRo to hear in noisy environments. }\end{array}$ \\
\hline Touch sensors & There are nine touch sensors that detect human touch using capacitance sensors: five on the body and four on the head. \\
\hline Status display & $\begin{array}{l}\text { LEDs on the chest indicate what PaPeRo is doing and how PaPeRo is feeling. LEDs on the mouth and cheeks indicate } \\
\text { its facial expressions. LEDs on the ears indicate when the robot hears human speech, and LEDs around the eyes } \\
\text { indicate when it sees people. The eye LEDs blink green when it is searching for people, and when it locates them, the } \\
\text { green light shines stably. When it sees a person's face, the green changes to orange. }\end{array}$ \\
\hline Battery & $\begin{array}{l}\text { Lithium-ion battery; continuous operation time: } \\
\text { Approximately } 110 \mathrm{~min} \\
\text { Battery charging time: } \\
\text { Approximately } 150 \mathrm{~min} \text { (when power is off) }\end{array}$ \\
\hline External interface & $\begin{array}{l}2 \text { USB ports (USB2.0) } \\
\text { Auxiliary microphone input } \\
\text { AV output (video, stereo audio) } \\
1 \text { Compact Flash slot (CFA Type I) } \\
\text { Display output (Analog RGB, mini D-sub } 15 \text { pins) } \\
\text { Power input (DC19.5V) } \\
\text { Self-charge power input }\end{array}$ \\
\hline
\end{tabular}
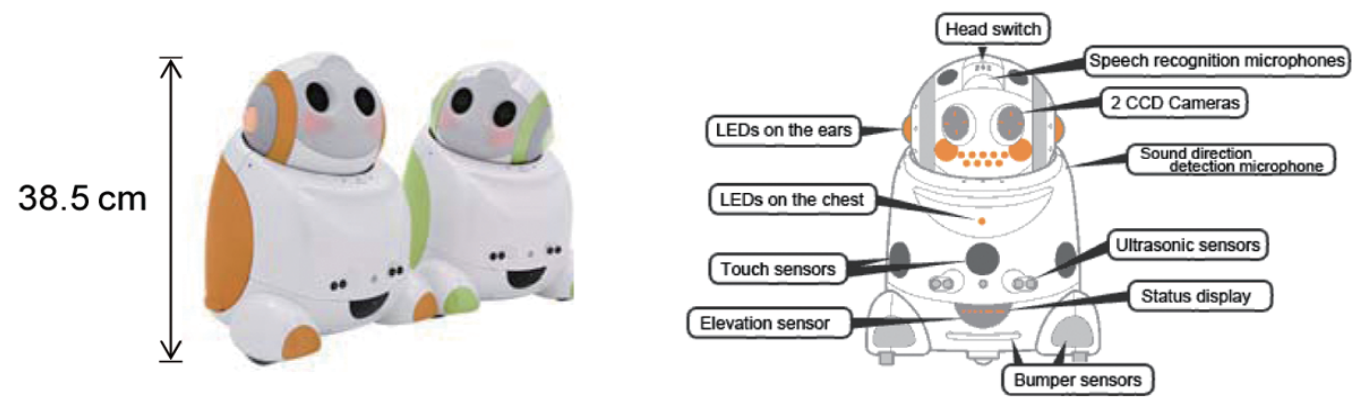

Fig. 1. PaPeRo(NEC) [14]. (Colours are visible in the online version of the article; http://dx.doi.org/10.3233/TAD-120357)

\section{Information support algorithm}

A method for interactive information support, based on the structure of the interaction between humans [11], was adopted for the following interactions: (1) the most basic way of starting a conversation, which involves an attention-seeking cue (an "alert" (a)), and (2) "presequence" information support, which facilitates the eventual exchange ("communication" (b)) that extends to the end of the conversation (Fig. 3).

(a) Alert: Owing to their diminished attention span, persons with dementia find it difficult to pay attention to the system at the beginning of a conversation. We have therefore proposed an interaction alert to direct their attention to the prototype, as a way to initiate a dialogue. 


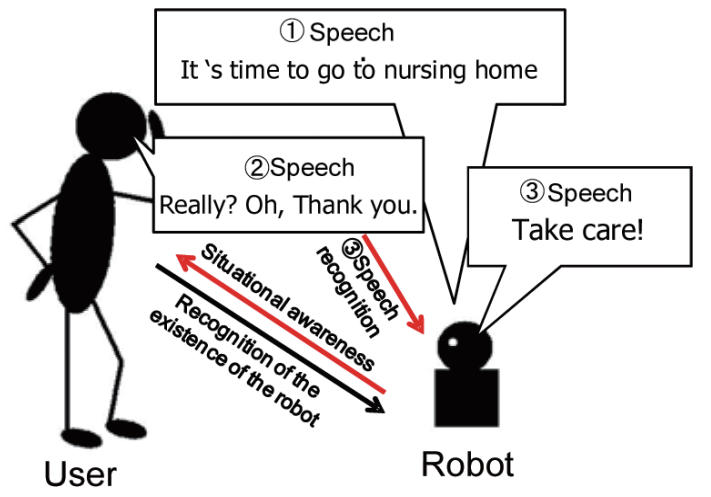

Fig. 2. Concept of the robot system. (Colours are visible in the online version of the article; http://dx.doi.org/10.3233/TAD-120357)

(b) Communication: The system recognizes whether its message has been cognized by the person with dementia, and it decides whether or not the information needs to be presented again. Therefore, a method for interactive communication is proposed.

We developed the first prototype as an interactive platform in order to implement the proposed method.

\section{Field-based development of information support robot}

\subsection{Development process}

We followed the field-based development process given below:

1) Select a cooperative user.

2) Observe the lifestyle of the user.

3) Interview the families and caregivers of the user.

4) Evaluate the physical and cognitive abilities of the user.

5) Identify the problems for which the robot needs to offer support.

6) Determine the support method and construct the robot system.

7) Install the first prototype into the user's home.

8) Identify problems and correct them.

9) Evaluate the attention function of the robot system.

10) Evaluate the information transmission function of the robot system.

11) Modify the prototype and build a second prototype.

\subsection{First prototype}

A 97-year-old woman with Alzheimer's disease was selected as a user who would cooperate with our project. Her cognitive deficit level was mild to middle (MiniMental State Examination (MMSE): 17, Clinical Dementia Rating Scale (CDR): 1). She lived alone independently in a special apartment for the elderly.

As a result of observation and interviews, it was identified that she had problems with remembering what time she went to the daytime nursing home. One problem was that she did not go to the door when a caregiver came to pick her up because she did not hear the doorbell. Another problem was that she sometimes forgot to go to the bathroom before leaving home, and was then incontinent on the courtesy bus that brought her to the nursing home. We therefore decided to focus on developing a robot that could offer support for these two activities.

Using an information support algorithm, we built the first prototype of the information support robot system for this cooperative user.

\subsection{Installation of the first prototype in the field}

The first prototype was installed in the user's home. Explanations, demonstrations, fitting, and training took place over five days. One of the problems encountered was that the user had difficulty understanding the synthesized speech of the robot. To remedy this, the speech of a male voice was recorded and was used instead of synthesized speech. After this modification, the user understood what the robot said and was able to acquire the required information from the robot.

Next, evaluation sessions were conducted over 5 days. The user talked with the robot for about one hour each day. The contents of the conversation were subjects of interest to the user that we learned from an earlier interview with her.

\subsection{Evaluation of the first prototype}

The attention-seeking function and the information distribution function were each evaluated for two hours a day, for seven days. The response rates $(\mathrm{Rr})$ for the evaluation of attention-seeking and the information acquisition rates (Ir) for the information distribution were calculated from the video data, as follows.

$$
\mathrm{Rr}=\frac{\mathrm{Nr}}{\mathrm{Nr}+\mathrm{Nn}} \times 100 \%
$$



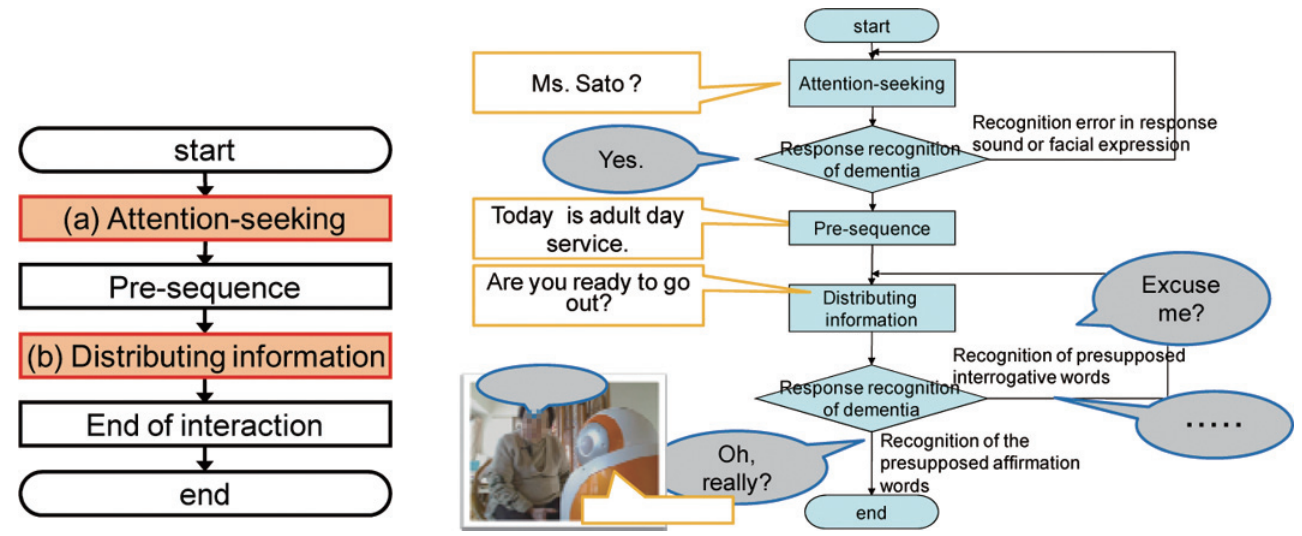

Fig. 3. Structure of interaction. (Colours are visible in the online version of the article; http://dx.doi.org/10.3233/TAD-120357)

Table 2

Results of the first prototype evaluation

\begin{tabular}{lcc}
\hline Rate & 1st distribution & $\begin{array}{c}\text { 1st }+2 \mathrm{nd} \\
\text { distribution }\end{array}$ \\
\hline $\mathrm{Rr}$ & $96.7 \%(59 / 61)$ & $96.7 \%(59 / 61)$ \\
$\mathrm{Ir}$ & $78.3 \%(54 / 69)$ & $94.2 \%(65 / 69)$ \\
\hline
\end{tabular}

${ }^{*}$ Rr: Response rate. ${ }^{*}$ Ir: Information acquisition rate.

where $\mathrm{Nr}$ is the number of responses to the robot's attention-seeking action, and $\mathrm{Nn}$ is the number of nonresponses to the robot's attention-seeking action.

$$
\mathrm{Ir}=\frac{\mathrm{Ni}}{\mathrm{Ni}+\mathrm{Nf}} \times 100 \%
$$

where $\mathrm{Ni}$ is the number of instances of information acquisition from the robot, and $\mathrm{Nf}$ is the number of failed instances of information acquisition from the robot.

Table 2 presents the results for $\mathrm{Rr}$ and $\mathrm{Ir}$. $\mathrm{Rr}$ was $96.7 \%$, as calculated from 61 attention-seeking actions. The reason for non-response was interruptions by the experimenter. Ir was 94.2\%, as calculated from 69 distributions of information. However, correct information was acquired from the first distribution of information only $78.3 \%$ of the time, and a second distribution was required $15.9 \%$ of the time. One of the problems encountered was speech recognition by the robot. There was wide variety of user responses to the robot, and the robot could not tell whether or not the user understood the information. This would then trigger a second round of information distribution from the robot.

\subsection{Second prototype}

As the result of the evaluation of the first prototype, we identified that there was a problem with the robot system's rate of speech recognition. In response to this discovery, we modified the information support algo-
Table 3

Profile of participants in evaluation of the second prototype

\begin{tabular}{ccllccc}
\hline Participant & Age & Gender & Disease & Care Level* $^{*}$ & MMSE & CDR \\
\hline A & 97 & Female & AD & 1 & 17 & 1 \\
B & 79 & Female & CVA & 1 & 17 & 2 \\
C & 83 & Female & AD like & 1 & 16 & 1 \\
D & 89 & Female & AD like & 1 & 17 & 1 \\
E & 81 & Female & AD & 1 & 23 & 1 \\
\hline
\end{tabular}

* Care Level shows the typical scale used in Japan with long term care insurance.

rithm for information distribution. We added a step involving confirmation for when the robot fails to recognize the user's response. Figure 4 shows the new algorithm that was developed. In this way, the robot was given a limited number of registered response words and had a higher recognition rate than it did previously.

\section{Evaluation of the second prototype}

\subsection{Participants and methods}

Validation of the communication method and an evaluation of the basic performance of the second prototype were performed in tests conducted with five persons with dementia, who were all living alone in nursing homes. The details of the five participants are provided in Table 3. All the participants were female, and the average and standard deviation of their age was $85.8 \pm 7.3$ years. Two participants had Alzheimer's disease (AD), two had Alzheimer's-disease-like symptoms (AD-like), and one participant had a cardiovascular accident (CVA). The care level, based on the scale used in Japanese long term care insurance, showed level 1 for all participants. Their scores on the MMSE ranged from 16 to 23 , and their scores on the CDR showed 1 


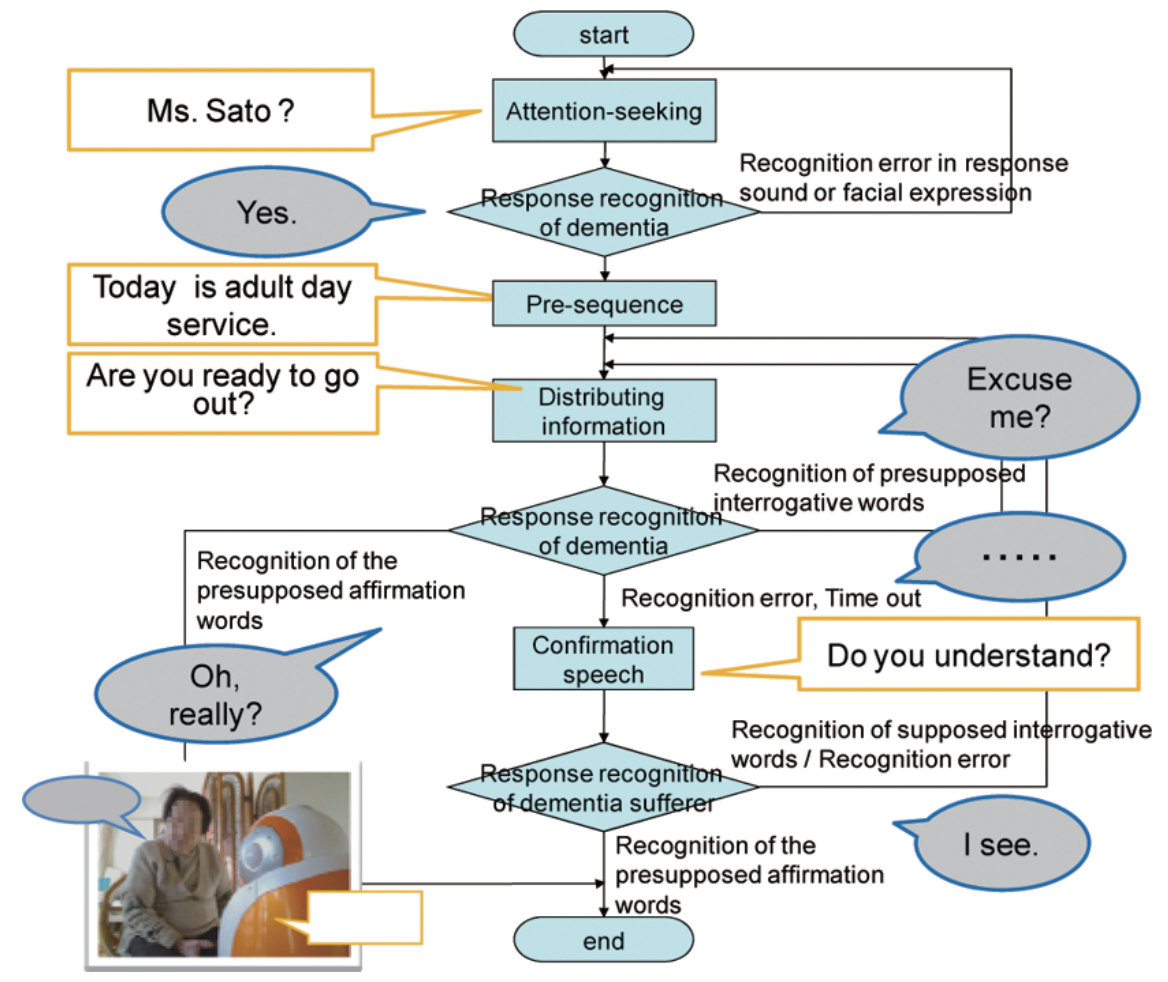

Fig. 4. Modified algorithms for information. (Colours are visible in the online version of the article; http://dx.doi.org/10.3233/TAD-120357)

for four participants, and 2 for one participant. Participant A was the same person as the cooperative user of the first prototype.

The evaluation methodology was based on the observation of the participants by a webcam and an IC recorder. The response rate $\mathrm{Rr}$ of the participants during the alert interaction was calculated using Eq. (1), and their information acquisition rate (Ir) during the communication interaction was calculated using Eq. (2).

\subsection{Experiment description}

We evaluated the alert and communication interactions of the participants. If the robot could not recognize the speech of a participant, the algorithm for the alert and communication interactions repeated the process - but only once. In order to improve the reliability of recognition, a closed question was used in response.

The evaluation sessions took place over 5 days for participant A, and 3 days for participants B, C, D, and E. Each day the participants talked with the robot for about $30 \mathrm{~min}$, covering content in their areas of interest.

In order to evaluate the robot's acceptability by the participants, their conversations in this regard were recorded and then documented as transcripts. The num- ber of their remarks about the acceptability of the robot was then counted.

\subsection{Experimental results}

The experiment with five participants showed a $100 \%$ rate of response to the alert interaction (Table 4). This result shows that the response rate for interaction with the prototype alert was high. As regards communication interaction, the rate of information acquisition was $90 \%$ or more for all subjects (Table 5). Participants $\mathrm{B}$ and $\mathrm{C}$ understood the information on the first distribution, for all the distributions. Participants A, C, and D failed twice on the first distribution, but understood the information on the second distribution. Participant A failed once with the second distribution. These results thus confirmed that the interaction of this communication was appropriate.

Table 6 presents the number of remarks made by the participants about the robot's acceptability, most of which were positive. Some typical phrases used to describe the robot were "pretty," "smart," and "great," and they commented on the fun of having conversation with a robot. Some of the subjects, on the other hand, expressed the difficulty they experienced in using the robot. 
Table 4

Response rates from second prototype evaluation

\begin{tabular}{ccc}
\hline Participant & $\operatorname{Rr}(1$ st distribution) & $\operatorname{Rr}(1$ st + 2nd distribution) \\
\hline A & $100.0 \%(26 / 26)$ & $100.0 \%(26 / 26)$ \\
B & $100.0 \%(15 / 15)$ & $100.0 \%(15 / 15)$ \\
C & $100.0 \%(15 / 15)$ & $100.0 \%(15 / 15)$ \\
D & $100.0 \%(15 / 15)$ & $100.0 \%(15 / 15)$ \\
E & $100.0 \%(14 / 14)$ & $100.0 \%(14 / 14)$ \\
\hline
\end{tabular}

* Rr: Response rate.

Table 5

Information acquisition rates from second prototype evaluation

\begin{tabular}{ccc}
\hline Participant & Ir (1st distribution) & Ir (1st + 2nd distribution) \\
\hline A & $84.6 \%(22 / 26)$ & $96.2 \%(25 / 26)$ \\
B & $100.0 \%(16 / 16)$ & $100.0 \%(16 / 16)$ \\
C & $86.7 \%(13 / 15)$ & $100.0 \%(15 / 15)$ \\
D & $87.5 \%(14 / 16)$ & $100.0 \%(16 / 16)$ \\
E & $100.0 \%(14 / 14)$ & $100.0 \%(14 / 14)$ \\
\hline
\end{tabular}

* Ir: Information acquisition rate

\subsection{Demonstration of life support (Case study)}

Within the evaluation session of the second prototype, we conducted a demonstration whose aim was to solve some problems in daily living experienced by participant $\mathrm{A}$, who was also the cooperative user of the first prototype, using the information support robot. Her problems were caused by not remembering the time she would be going to the daytime nursing home (as was mentioned in 3.2.). To solve these problems, we created contents to provide information that consisted of the following two consecutive steps:

1) A prompt to go to the bathroom $10 \mathrm{~min}$ before it was time to leave home for the daytime nursing home.

2) A prompt to go to the entrance when the doorbell rang.

These prompts were provided for 3 days. The total number of information prompts was 6: 3 times for the bathroom and 3 for the doorbell.

The results showed three kinds of behavior by the user: (1) movement in response to the robot's prompt (3 times), (2) not being able to move in response to the robot's prompt (2 times), and (3) not moving in response to the robot's prompt of her own free will (1 time). The second kind of behavior was caused either by her attention wandering after she began to move, or by doubt regarding the prompt from the robot. The cause of the third kind of behavior was both interesting and significant. She had already gone to the bathroom just prior to the robot's prompt. Upon hearing the prompt, she simply answered, "I've already gone to the bathroom." This reply suggests that the support from the robot improved the autonomy of a person with dementia, who was encouraged to act from her own will.

\section{Discussion}

\subsection{Effectiveness of the information support robot}

The results of the information acquisition experiment using the second prototype revealed that the information support robot could provide important information for its users' daily life. The three-step structure of the interaction that involved attention-seeking, a presequence, and the distribution of information worked well in providing the desired information to persons with mild to intermediate dementia. The function of the robot that repetitively provides information is also important, as it increased the information acquisition rate (Ir).

The results of the life support demonstration were amazing because they even implied that the robot could prompt behaviors of a person with dementia. They also demonstrated that the information support provided by the robot also has the potential to improve their autonomy. This can be considered as one of its major advantages over human support.

Therefore, the results suggested the effectiveness and usefulness of the information support robot in providing assistance in the daily life of persons with a mild level of dementia.

\subsection{Efficacy of the field-based method}

One point worth emphasizing is the modification of the robot, whose synthesized speech was changed to recorded male speech in response to a need that was identified in the field evaluation of the first prototype. Although this kind of problem is widely known, detailed data are not available. Although we must obtain more data to clarify the characteristics of this issue, we would emphasize here that the identification of this problem was the result of field-based development. A second point worth emphasizing is the problem of speech recognition by the robot, which was also revealed in the field evaluation of the first prototype. From a technical point of view, we needed to try to modify the speech-recognition engine. We tried, however, to find a solution based on a field-based point of view, and it turned out that a change in the informa- 
Table 6

Acceptability of the robot

\begin{tabular}{|c|c|c|c|}
\hline \multirow[t]{2}{*}{ Subject } & \multicolumn{2}{|c|}{ No. of remarks about acceptability } & \multirow[t]{2}{*}{ Examples of remarks } \\
\hline & Positive & Negative & \\
\hline A & 14 & 2 & $\begin{array}{l}\text { P: "You are pretty." "You are smart." "I enjoyed the conversations with you." } \\
\text { N: "I don't need it." "It is difficult to use it." }\end{array}$ \\
\hline B & 0 & 0 & - \\
\hline $\mathrm{C}$ & 6 & 1 & $\begin{array}{l}\text { P: "You are pretty." "You are smart." "I enjoyed the conversations with you." "There is no } \\
\text { oppressive feeling." } \\
\text { N: "Children must like it." }\end{array}$ \\
\hline $\mathrm{D}$ & 6 & 0 & P: "This is great." "I enjoyed the conversation." "Please come again." \\
\hline $\mathrm{E}$ & 1 & 1 & $\begin{array}{l}\text { P: "This is great." } \\
\text { N: "I must learn the mechanism if I use it." }\end{array}$ \\
\hline
\end{tabular}

tion support algorithm effectively increased the speech recognition rate by simplifying the user's answers.

Through this development, the effectiveness of the field-based method was confirmed. A robot is one example of possible technologies that could be employed. It is our view that additional technologies, which include advanced technologies, must be involved in the field of assistive technology for persons with dementia. Field-based development and innovation have high potential to match users' needs with an appropriate technology, and to thus improve the independence, autonomy, participation, and dignity of persons with dementia.

\subsection{Limitations and future work}

One of the limitations of this study is that this development is still in a very nascent stage. Validation of the field-based method that we propose will require more progress in the areas of development and evaluation. However, there need not be not only a single large-scale development and evaluation cycle. Smaller cycles of development and evaluation are also important, as we know from the COGKNOW project, which adopted three small cycles. This study, then, represents the implementation of its first small cycle, which has already produced significant results.

Dementia sufferers display a complicated variety of symptoms. We conducted our evaluation with only 5 participants and our demonstration with 1 participant, which of course limits the statistical authority of the results. In order to more conclusively prove the effectiveness of the information support robot, more data will need to be collected. Our intention is to conduct further experiments that we hope will show the robot's effectiveness and the validity of its application to different types and levels of dementia.

Future works will involve more field tests and modification of the system in order to build a more practical and useful robot system. The PaPeRo system has a mobility function, vision sensor, as well as other functions, and our aim is to match these functions with users for whom they are suitable.

In addition, workshops that include stakeholders, users, caregivers, therapists, developers, researchers, and other professionals will be planned. Field tests will reveal a suitable system concept for each user. Sometimes, the results may indicate the need for some degree of trade-off among users. A workshop is an effective means of achieving consensus among stakeholders regarding a system concept, and we intend to employ this approach for this purpose. In the final stage of this project, we are also planning a social experiment that will confirm the effectiveness of the overall large cycle, using field-based development and innovation. This large cycle will represent a novel comprehensive package that involves the development of state-of-theart assistive technologies.

\section{Conclusions}

In this study, we developed two prototypes of an information support robot for persons with dementia, using field-based methodology. The communication robot system produced by NEC Corporation, called "PaPeRo", was chosen as the platform for this system. To achieve the goal of keeping persons with dementia informed of their daily schedule and prompting them into action, interactive verbal communication algorithms were programmed into the robot system. The results of experiments conducted with five elderly women with dementia showed that the robot system produced an information acquisition rate of over $90 \%$. Next, a life support demonstration confirmed that the robot could successfully prompt the users to take action. In addition, these results suggested the possibility of using this robot to support independent living by persons with dementia. As another result of this development, the effectiveness of the field-based method was confirmed. 


\section{Acknowledgements}

We would like to thank Seikatsu Kagaku Un-Ei Co., Ltd. This research was partially supported by the Japan Foundation for Aging and Health, KAKENHI (21300213), and the Japan Science and Technology Agency, JST, under its Strategic Promotion of Innovative Research and Development Program.

\section{References}

[1] World Health Organization (WHO) and Alzheimer's Disease International, Dementia: A public health priority, WHO, (2012).

[2] The Ministry of Health, Labor and Welfare of Japan, Report of Care for the Elderly Policy Committee "Care for the Elderly in 2015", http://www.mhlw.go.jp/topics/kaigo/kentou/15kourei/ 3.html (2003). (in Japanese).

[3] The Ministry of Economy, Trade and Industry of Japan, Report of Robot Industry Policy Committee, http://www.meti. go.jp/press/20090325002/20090325002-3.pdf (2009). (in Japanese).

[4] A. Mihailidis and G. Fernie, Context-aware devices for older adults with dementia, Gerontechnology, Vol. 2 (2002), 173189.

[5] M. Bourgeois, Enhancing conversation skills in patients with Alzheimer's disease using a prosthetic memory aid, J Appl Behav Anal, Vol. 23, No. 1 (1990), 29-42.

[6] M. Hoffman, C. Hock, A. Kühler and F. Müller-Spahn, In- teractive computer-based cognitive training in patients with Alzheimer's disease, J. Psychiatr. Res., Vol. 30, No. 6 (1996), 493-501.

[7] M. Oriani, E. Moniz-Cook, G. Binetti, G. Zanieri, G. Frisono, C. Geroldi, L. De Vreese, and O. Zanetti, An electronic memory aid to support prospective memory in patients in the early stages of Alzheimer's disease: A pilot study, Aging Ment Health, Vol. 7, No. 1 (2003), 22-27.

[8] R. Levinson, The planning and execution assistant and training system, J Head Trauma Rehabil, Vol. 12, No. 2 (1997), 769775 .

[9] M. Pollack, L. Brown, D. Colbry, C. McCarthy, C. Orosz, B. Peintner, S. Ramakrishnan and I. Tsamardinos, Autominder: An intelligent cognitive orthotic system for people with memory impairment, Robot Auton Syst, Vol. 44 (2003), 273-282.

[10] The MonAMI Project, http://www.monami.info/, (2011).

[11] S. Bjoerneby, P. Duff and O. Maki, Developing assistive technology for people with dementia, Assistive Technology Research Series Vol. 11, Assistive Technology - Shaping the Future AAATE2003 (2003), 781-786.

[12] Ed. by R. Castellot, COGKNOW Final Report: WP 0 Project Management, (2009).

[13] T. Inoue, R. Ishiwata, R. Suzuki, T. Narita, M. Kamata, M. Shino and M. Yaoita, Development by a field-based method of a daily-plan indicator for persons with dementia, Assistive Technology Research Series Vol. 25, Assistive Technology from Adapted Equipment to Inclusive Environments AAATE2009 (2009), 364-368.

[14] NEC, Communication Robot PaPeRo, http://www.nec.co.jp/ products/robot/en/index.html, (2012).

[15] S. Okamoto, Social Psychology of Language, 3rd Ed., Nakanishiya Shuppan, Kyoto, Japan (2006). (in Japanese) 